\title{
Perfil de ácidos graxos e composição centesimal de carpas (Cyprinus carpio) alimentadas com ração e com dejetos suínos
}

\author{
Fatty acid profile and proximate composition of carp (Cyprinus carpio) feed artificial food and pig manure
}

\author{
Janice Izabel Druzian $^{\mathrm{I} \text {,II }}$ Cristiane Michele Marchesi ${ }^{\mathrm{I}}$ Adilma Regina Pippa Scamparini ${ }^{\mathrm{III}}$
}

\section{RESUMO}

Este estudo investigou o efeito da alimentação com dejetos suínos na composição centesimal e no perfil de ácidos graxos do músculo de carpas. Amostras de carpas com 4-10 meses de idade, recolhidas em Santa Catarina, foram filetadas, trituradas e acondicionadas em atmosfera de nitrogênio $\left(-18^{\circ} \mathrm{C}\right)$. Os valores (\%) de umidade, proteína e cinzas foram: 72,$24 ; 14,85$; e 2,16, respectivamente, para carpas alimentados com ração; e 83,33; 14,48; e 3,49 para carpas alimentadas com dejetos suínos. Os valores de sódio e potássio foram: 0,491\% e 1,20\% carpas/ração; e 2,28\% e 0,367\% carpas/dejetos suínos, respectivamente. Os teores de lipídios totais também apresentaram diferenças significativas: 9,88\% carpas/ração e 1,69\% carpas/dejetos suínos. O perfil de ácidos graxos mostrou tendências similares nos dois músculos. Portanto, verificou-se um efeito significativo da alteração da dieta sobre a composição do tecido muscular de carpas alimentadas com dejetos suínos, principalmente no conteúdo de minerais e de lipídios totais, embora a variação observada no conteúdo de lipídios totais não tenha se refletido na composição de ácidos graxos.

Palavras-chave: composição centesimal, perfil de ácidos graxos, carpas (Cyprinus carpio), alimentadas com dejetos suínos.

\section{ABSTRACT}

This study investigated the proximate composition and fatty acid profile of the carp muscle fed with pig manure. Carp with 4-10 months of age were collected in Santa Catarina. Muscle samples were sliced and then triturated in a multiprocessor and stored under gaseous nitrogen atmosphere $\left(-18^{\circ} \mathrm{C}\right)$. The moisture, protein, and ash contents (\%) were as follows: 72.24; 14.85; e 2.16, respectively, for carp feed artificial food; and 83.33; 14.48; e 3.49 for carp feed pig manure. The contents of sodium and potassium were: $0.491 \%$ and $1.20 \%$ carp/artificial food; $2.28 \%$ and $0.367 \%$ carp/pig manure. The total lipids contents also presented significant differences: 9.88\% carp/artificial food, and $1.69 \%$ carp/pig manure. Fatty acid profile showed similar tends in both muscles. Therefore, a significant difference in the total lipids and mineral contents of the carp tissue feed pig manure was detected, though the observed variation in total lipid content wasn't reflected in the fatty acid composition.

Key words: proximate composition, fatty acid profile, carp (Cyprinus carpio), feed pig manure.

\section{INTRODUÇÃO}

O desenvolvimento da suinocultura trouxe como conseqüência a intensa produção de dejetos, os quais são altamente poluentes e causadores da degradação ambiental. De acordo com dados do Instituto Brasileiro de Geografia e Estatística, o rebanho nacional em 2004 era de aproximadamente 33 milhões de suínos (IBGE, 2004). Segundo estimativas, esta produção gera de 32 a 51 milhões de toneladas de dejetos/ano, sendo Santa Catarina responsável por 10\% deste montante, dos quais aproximadamente $70 \%$ estão concentrados na região Oeste do Estado, onde grande parte do resíduo é utilizado na alimentação de outros animais e como fertilizante do solo (CAVALCANTI, 1984).

ICentro de Ciências Agroambientais, Universidade do Oeste de Santa Catarina (UNOESC), CP 187, 89560-000, Videira, SC, Brasil.

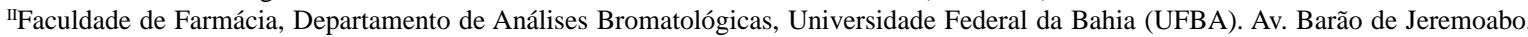
s/n, Ondina, 40171-970, Salvador, BA, Brasil. E-mail: druzian@ufba.br.

IIIFaculdade de Engenharia de Alimentos, Departamento de Ciências de Alimentos, Universidade Estadual de Campinas (UNICAMP), CP 6121, 13081-970, Campinas, SP, Brasil. 
Estudos realizados sobre índices de conversão alimentar orientam sobre o aproveitamento racional dos dejetos de suínos na alimentação e engorda de animais, o que contribui para a produção de carne a baixo custo (OLIVEIRA et al., 2002; BARRETO et al., 2004a; BARRETO et al., 2004b).

A prática de utilizar a matéria orgânica de dejeto suíno para alimentação de várias espécies de peixes é comum principalmente na Região Oeste Catarinense. Utilizando-se matéria orgânica de dejetos, consegue-se suprir as necessidades nutritivas das várias espécies de carpas (DIGGS et al., 1965; SILVA et al., 1987; TOMPSON, 1997; KOOP, 1996); entretanto, não existem dados comparando a composição centesimal e de ácidos graxos do tecido muscular de peixes submetidos à dieta com dejetos suínos. A utilização de dejetos na alimentação de peixes é comum também em vários países do mundo. Como exemplo, tem-se o cultivo da carpa chinesa, prateada, cabeça grande, capim e carpa comum. A matéria orgânica de dejetos com minerais consegue suprir as necessidades nutritivas das várias espécies de carpas (KOOP, 1996; TOMPSON, 1997).

Lipídios de alimentos contêm uma grande variedade de ácidos graxos, diferindo na cadeia lateral, no grau de insaturação, na posição e configuração das duplas ligações, na presença de grupos funcionais especiais e nos isômeros de posição e geométricos. Tipicamente, lipídios de peixes contêm ácidos graxos com cadeia lateral que variam de 14 a 22 carbonos e com 0-6 metilenos interrompidos por duplas ligações (KRZYNOWERK \& PANUNZIO, 1989; KING et al., 1990).

Estudos clínicos e epidemiológicos na última década indicam que o consumo de peixes e óleos de peixes pode melhorar ou prevenir certas condições cardiovasculares humanas (MCCOLL, 2003; SIMOLOULOS, 2003; SCHACKY \& VON, 2004). Apesar do consenso das pesquisas sobre o efeito benéfico da ingestão de peixes e das doenças cardiovasculares serem a maior causa de morte no Brasil, dados da composição dos peixes produzidos no país ainda são escassos.

É importante avaliar essa propriedade terapêutica potencial em termos da determinação do conteúdo de ácidos graxos, assim como a composição nutricional de peixes produzidos no Brasil e submetidos a dietas diferenciadas. Portanto, o objetivo deste trabalho foi avaliar a influência na composição centesimal e de ácidos graxos de carpas alimentadas com dejetos suínos, quando comparadas a carpas alimentadas com ração. Estudos foram especialmente concentrados na determinação apurada do conteúdo de ácidos graxos de interesse médico.

\section{MATERIAL E MÉTODOS}

As amostras das carpas foram recolhidas em açudes situados na região Oeste de Santa Catarina, mais especificamente no vale do rio do Peixe e no Sul do Estado, no mesmo período do ano. Foram recolhidas, de modo representativo, 12 amostras de carpas comuns (Cyprinus carpio) alimentadas com dejetos de suínos e 12 alimentadas com ração. As carpas apresentavam idades entre 4 a 10 meses e peso entre 200-700g. As amostras foram filetadas, parte liofilizada, e acondicionadas a $-18^{\circ} \mathrm{C}$ em atmosfera de $\mathrm{N}_{2}$ gasoso. A parte filetada foi usada para análise da composição centesimal e mineral, e a parte liofilizada para análise da composição de ácidos graxos. Foi analisado o tecido muscular das amostras e todas as determinações foram realizadas em triplicatas.

Os métodos utilizados para as determinações de umidade, matéria seca, cinza, e proteína bruta foram os descritos na AOAC (2000). Minerais (Na e $\mathrm{K}$ ) foram determinados a partir das cinzas por fotometria de chama, e a quantificação foi realizada pelo uso de padrão externo com soluções padrões de $\mathrm{NaCl}$ e $\mathrm{KCl}$ (Merck) (AOAC, 2000). O conteúdo de lipídios foi determinado pelo método de BLIGH\&DYER(1959).

Para a determinação da composição dos ácidos graxos, os lipídios extraídos foram saponificados conjuntamente, e os ácidos graxos totais resultantes foram metilados de acordo com MAIA (1994). A identificação foi realizada por comparação dos tempos de retenção (tr) dos picos das amostras com os tr dos padrões (Sigma, St. Louis, MO, USA), e o espectro de massa de cada componente foi comparado aos da biblioteca Wiley. A quantificação foi realizada por normalização.

O cromatógrafo CG 17A / QP - 5000 (Shimadzu), com coluna DB-5 (30m x 0,22mm Hicap), foi utilizado, com temperatura do injetor e detector de 260 e $280^{\circ} \mathrm{C}$, respectivamente, pressão da coluna 120(Kpa), fluxo do Helio 0,8ml mim-1, programa de temperatura $\left(140-180^{\circ} / 1^{\circ} \mathrm{C}\right.$ por minuto, $180-280^{\circ} \mathrm{C} / 0,8^{\circ} \mathrm{C}$ por minuto, permanecendo a $280^{\circ} \mathrm{C}$ por 5 minutos). $\mathrm{O}$ intervalo $(\mathrm{m} / \mathrm{z})$ do detector EM foi de 40 à 650, com ganho de $1,50 \mathrm{KV}$.

Os principais equipamentos utilizados foram: Fotômetro de Chama Micronal (B 462), Mufla Quimis, Estufa Fanem (315 SE), determinador de proteína Kjedhal Tecnal (TE 036) e Liofilizador (CRYODOS-80). A comparação dos valores médios dos parâmetros avaliados nos músculos das carpas submetidas às duas dietas foi efetuada por meio de Análise de Variância ANOVA(Planilha Excel 2003), considerando um nível de significância de $5 \%(\mathrm{P}<0,05)$. 


\section{RESULTADOS E DISCUSSÃO}

Os valores (\%) obtidos para umidade, proteína, lipídios totais e cinzas relativos à amostra integral dos tecidos de carpas alimentadas com ração representam $73,13 \mathrm{cal} 100 \mathrm{~g}^{-1}$. Para tecidos de carpas alimentadas com dejetos suínos, este valor é de

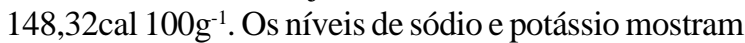
que a alimentação de carpas com dejetos suínos altera significativamente a composição mineral. Os teores de lipídios totais também apresentaram diferenças significativas (tcalc $(9,32)>>$ ttab $(2,26)$ para Test- $t$ bicaudal) entre os dois tipos de amostras. Valores médios de 9,88 \% $( \pm 3,03)$ foram encontrados para amostras de carpas alimentadas com ração e de 1,69 \% $( \pm 0,33)$ para carpas alimentadas com dejetos suínos. Os valores de desvios-padrão entre amostras foram altos, principalmente para lipídios totais e para cinzas, mostrando grandes variações no conteúdo dos nutrientes examinados, influenciados pela idade, mas principalmente pela dieta das carpas (Tabela 1 ).

Resultados bastante variados também foram obtidos por GURGEL \& FREITAS (1972), ao determinar a composição de 12 espécies de peixes de açudes do Nordeste brasileiro durante um ano. A matéria seca nas doze espécies durante o período variou de 21,9 a $41,4 \%$. Os teores de proteína variaram de 16,0 a $21,1 \%$, enquanto as médias dos teores de gorduras entre espécies e dentro de espécies variaram de 1,1 a 24,6\%.

Essas variações estão estreitamente relacionadas com a alimentação, uma vez que, durante os períodos de intensa alimentação, o conteúdo de proteínas do músculo aumenta no início muito lentamente, enquanto os conteúdos dos lipídios mostram um rápido e forte aumento. Os fatores naturais, como escassez de alimentos, ou fisiológicos, como desova ou migrações, influenciam também na composição química, sendo a fração lipídica a que mais apresenta variações (GURGEL \& FREITAS, 1972;
KRZYNOWERK \& PANUNZIO, 1989; KING etal., 1990; MAIA et al., 1994). O efeito da temperatura da água na composição de ácidos graxos também tem sido relatado. A proporção dos ácidos graxos insaturados tende a aumentar quando a temperatura diminui como de clima frio. A extensão em que esta tendência ocorre varia com a espécie e o tecido do peixe (MAIA et al., 1983).

Os lipídios totais foram saponificados e a composição de ácidos graxos metilados resultantes foram analisados por cromatografia gasosa com detecção de espectrometria de massas (CG-EM) (Figura 1). A tabela 2 apresenta os principais ésteres de ácidos graxos de carpas alimentadas com ração e de carpas alimentadas com dejetos suínos (Correlação Pearson = 0,9384 e tcrítico uni-caudal $=1,69$ para Test-t com amostras pareadas). Os valores obtidos de C16:0; C16:1n7; C17:0; C18:0; C18:1n9; C18:1n7 e C18:2n4 somados representam aproximadamente $70 \%$ dos ácidos graxos de carpas alimentadas com ração e $74 \%$ dos ácidos graxos de carpas alimentadas com dejetos. Os dados da composição de ácidos graxos, agrupados em séries de saturados, monoinsaturados, polinsaturados e com relação aos que apresentam atividade terapêutica 20:5 w3 e 22:6w3 (EPA e DHA), estão apresentados na tabela 3 . As diferenças entre os grupos de ácidos graxos não foram significativas (Pvalue $<<0,05)$. Carpas (Cyprinus carpio), independentemente da fonte de alimentação, apresentam baixa quantidade de EPA e DHA. Entretanto, como a quantidade de lipídios totais em carpas alimentadas com dejetos suínos é menor, as quantidades de EPA e DHA também serão menores, uma vez que a normalização dos ácidos graxos é relativa à porcentagem total de lipídios.

Nutricionalmente, quanto maior o valor de polinsaturados melhor. Os estudos mostram que a relação entre N-3 e N-6 fornece indicações importantes, considerando que $\mathrm{N}-3$ é benéfico à saúde, enquanto N-6 não tem a mesma correlação (MAYSER et al., 1998;

Tabela 1 - Composição química (\%) do tecido muscular de carpas alimentadas com dejetos suínos e de carpas alimentadas com ração.

\begin{tabular}{|c|c|c|c|c|c|c|c|}
\hline \multicolumn{2}{|c|}{ Dieta alimentar } & \multirow{2}{*}{$\begin{array}{c}\text { Umidade } \\
83,33\end{array}$} & \multirow{2}{*}{$\begin{array}{c}\text { Proteínas } \\
14,48\end{array}$} & \multirow{2}{*}{$\frac{\text { Lipídios Totais }}{1,69}$} & \multirow{2}{*}{$\begin{array}{c}\text { Cinzas } \\
3,49\end{array}$} & \multirow{2}{*}{$\begin{array}{c}\text { Sódio } \\
2,28\end{array}$} & \multirow{2}{*}{$\begin{array}{r}\text { Potássio } \\
0,3670\end{array}$} \\
\hline & M & & & & & & \\
\hline \multirow[t]{3}{*}{ Dejetos suínos } & $\mathrm{S}$ & 0,8136 & 0,0936 & 0,3350 & 0,2832 & 0,0739 & 0,0150 \\
\hline & CV(\%) & 0,9764 & 0,6465 & 19,83 & 8,12 & 3,25 & 4,09 \\
\hline & M & 72,24 & 14,85 & 9,88 & 2,16 & 0,4910 & 1,20 \\
\hline \multirow[t]{2}{*}{ Ração } & $\mathrm{S}$ & 2,85 & 2,29 & 3,03 & 0,2323 & 0,0179 & 0,0339 \\
\hline & CV(\%) & 3,95 & 15,43 & 30,63 & 10,76 & 3,64 & 2,82 \\
\hline
\end{tabular}

M=Média de análises em triplicatas de 12 amostras de tecido muscular.

$\mathrm{S}=$ Desvio padrão entre amostras. $\mathrm{CV}=$ Coeficiente de variação entre amostras.

Ciência Rural, v.37, n.2, mar-abr, 2007. 

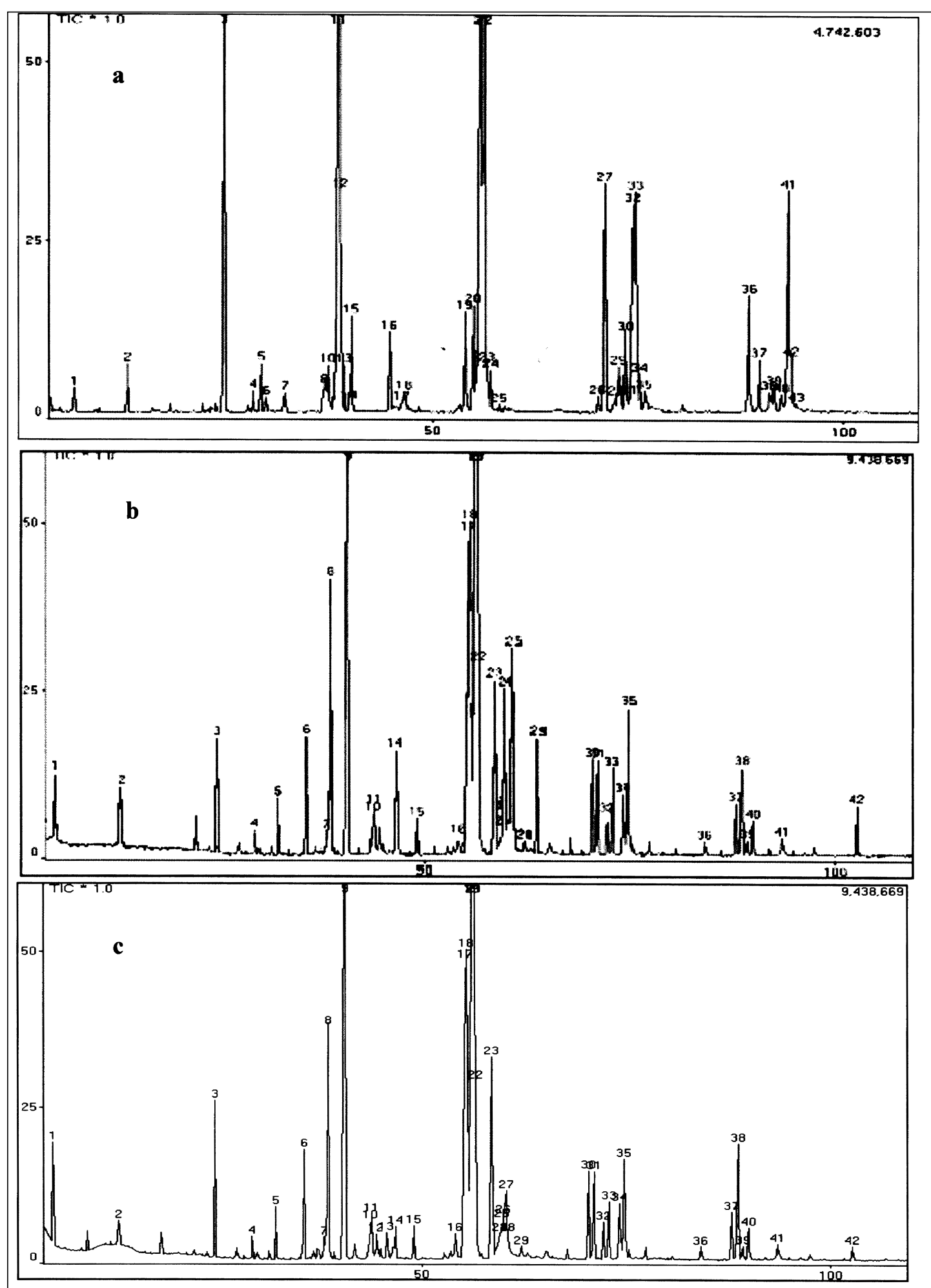

Figura 1 - Cromatogramas de ésteres de ácidos graxos obtidos por Cromatografia Gasosa - Espectrometria de massas (CG-EM): padrões (a), carpas alimentadas com dejetos de suínos (b), e carpas alimentadas com ração (c). ColunaDB-5 (140-180\% $\left.1^{\circ} \mathrm{C} \min , 180-280^{\circ} \mathrm{C} / 0,8^{\circ} \mathrm{C} \min , 280^{\circ} \mathrm{C} / 5 \mathrm{~min}\right)$, e $0,8 \mathrm{~mL} \mathrm{~min}{ }^{-1}$ de Hélio. Identificação dos picos na tabela 2 . 
Perfil de ácidos graxos e composição centesimal de carpas (Cyprinus carpio) alimentadas com ração...

Tabela 2 - Identificação e quantificação de ácidos graxos de carpas alimentadas com ração e com de dejetos de suínos, determinados por CG$\mathrm{EM}^{*}$. (NI=não identificado).

\begin{tabular}{|c|c|c|c|c|}
\hline \multirow{2}{*}{$\operatorname{Tr}(\min )$} & \multirow{2}{*}{ Ácidos graxos } & \multicolumn{3}{|c|}{$\%$ Ácidos Graxos \pm dp (número do pico relativo ao cromatograma da Figura 1 ) } \\
\hline & & Padrões & Carpas/Ração & Carpas/Dejetos \\
\hline 5,06 & hexanodióico & - & $0,49 \pm 0,01(1)$ & $1,00 \pm 0,01(1)$ \\
\hline 9,20 & C8:0 & - & - & - \\
\hline 11,94 & C10:0 & - & - & $1,30 \pm 0,02(2)$ \\
\hline 13,05 & $\mathrm{C} 12: 0$ & $0,51(2)$ & $0,31 \pm 0,01$ & - \\
\hline 21,23 & C13:0 & - & - & $0,38 \pm 0,02$ \\
\hline 24,73 & C14:0 & $9,08(3)$ & $2,00 \pm 0,01(3)$ & $1,19 \pm 0,01(3)$ \\
\hline 32,13 & C15:0 & $0,37(7)$ & $0,77 \pm 0,01$ (5) & $0,33 \pm 0,01(5)$ \\
\hline 33,08 & C15:1n9 & - & - & $1,10 \pm 0,01(6)$ \\
\hline 38,78 & C16:0 & $17,48(11)$ & $4,32 \pm 0,04(8)$ & $6,71 \pm 0,05(8)$ \\
\hline 40,71 & C16:1n7 & $1,43(12)$ & $15,45 \pm 0,18(9)$ & $16,80 \pm 0,20(9)$ \\
\hline 46,13 & $\mathrm{C} 16: 2 \mathrm{n} 7$ & $1,36(16)$ & $0,59 \pm 0,01(14)$ & $1,35 \pm 0,01(14)$ \\
\hline 55,34 & C17:0 & 1,28 (19) & $9,90 \pm 0,08$ (17) & $5,78 \pm 0,10(17)$ \\
\hline 55,41 & C17:1n11 & - & $4,75 \pm 0,13(18)$ & $1,37 \pm 0,12(18)$ \\
\hline 55,53 & C16:4n3 & - & - & $1,04 \pm 0,03$ (19) \\
\hline 55,57 & C18:1DMA & - & - & $1,08 \pm 0,08(20)$ \\
\hline 56,28 & C18:0 & $18,8(21)$ & $13,34 \pm 0,39(21)$ & $15,08 \pm 0,32(21)$ \\
\hline 56,40 & C18:1n9 & $2,60(22)$ & $15,40 \pm 0,38(22)$ & $15,58 \pm 0,42(22)$ \\
\hline 56,69 & C18:1n7 & 3,67 (23) & $6,63 \pm 0,031(23)$ & $4,48 \pm 0,024(23)$ \\
\hline 56,76 & C18:2n6 & 8,67 (24) & $2,75 \pm 0,08$ (25) & $4,19 \pm 0,11(25)$ \\
\hline 58,69 & C18:2n4 & $0,81(25)$ & $4,73 \pm 0,12(27)$ & $6,15 \pm 0,16(27)$ \\
\hline 59,60 & C18:3n6 & 0,97 & $1,12 \pm 0,08$ (28) & $0,55 \pm 0,07$ (28) \\
\hline 60,38 & C18:3n3 & - & $2,56 \pm 0,09$ (29) & $2,30 \pm 0,08$ (29) \\
\hline 70,52 & C18:4n3 & $0,48(26)$ & $2,04 \pm 0,06(30)$ & $1,78 \pm 0,08(30)$ \\
\hline 71,15 & C20:0 & 6,97 (27) & $1,96 \pm 0,12(31)$ & $0,85 \pm 0,15(31)$ \\
\hline 72,30 & C20:1n9 & - & $0,88 \pm 0,09(32)$ & $0,57 \pm 0,08(32)$ \\
\hline 72,99 & C20:2n6 & $1,57(30)$ & $1,32 \pm 0,07$ (33) & $0,51 \pm 0,06$ (33) \\
\hline 74,26 & C20:4n6 & 2,19 (31) & $1,16 \pm 0,05$ (34) & $1,25 \pm 0,09$ (34) \\
\hline 74,87 & C20:5w3 (EPA) & $6,74(32)$ & $2,44 \pm 0,09$ (35) & $3,15 \pm 0,06$ (35) \\
\hline 75,20 & C 22:0 & $4,72(33)$ & - & - \\
\hline 84,29 & C 22: ?n3 & - & $0,30 \pm 0,07$ (36) & $0,47 \pm 0,04(36)$ \\
\hline 88,03 & C 22:5n3 & - & $1,10 \pm 0,06$ (37) & $1,03 \pm 0,05$ (37) \\
\hline 88,71 & C 22:6 w3 (DHA) & $3,40(36)$ & $2,92 \pm 0,04$ (38) & $1,02 \pm 0,07$ (38) \\
\hline 93,64 & $\mathrm{C} 24: 0$ & $6,90(41)$ & - & - \\
\hline 101,87 & NI & - & $0,40 \pm 0,05(42)$ & $1,21 \pm 0,07(42)$ \\
\hline 102,71 & NI & - & $0,37 \pm 0,04$ & $0,34 \pm 0,07$ \\
\hline TOTAL & & 100,00 & 100,00 & 100,00 \\
\hline
\end{tabular}

${ }^{*}$ Cromatografia Gasosa - Espectrometria de massas.

ROSE \& CONNOLLY, 1999; MCCOLL, 2003). Carpas alimentadas com ração possuem uma razão entre N-3/ N-6 menor, se comparada com as carpas alimentadas com dejetos suínos. Os ácidos graxos EPA e DHA possuem atividade terapêutica na prevenção de doenças (MAYSER et al., 1998; ROSE \& CONNOLLY, 1999; MCCOLL, 2003; SCHACKY \& VON SCHACKY, 2004). A investigação da composição química, particularmente com relação à composição de ácidos graxos e colesterol no conteúdo lipídico de peixes, tem sido objetivada pela comunidade cientifica mundial, por estar relacionada diretamente a saúde humana. Dentre os ácidos graxos, os pertencentes à família ômega-3, como o ácido alfa-linolênico (LNA, 18:3w3), e, particularmente, os ácidos eicosapentaenóico (EPA, 20:5w3) e o docosahexaenóico (DHA, 22:6w3), têm recebido maior atenção por reduzirem fatores de risco associados às doenças cardiovasculares, psoríase, 
Tabela 3 - Valores obtidos (\%) para as diferentes séries de ácidos graxos totais de carpas, de acordo com a dieta alimentar.

\begin{tabular}{lcc}
\hline \multirow{2}{*}{ Grupos de ácidos graxos } & \multicolumn{2}{c}{ Dieta alimentar } \\
\cline { 2 - 3 } & Ração & Dejetos suínos \\
\hline Saturados & 33,09 & 32,55 \\
Monoinsaturados & 43,12 & 43,21 \\
Polinsaturados & 23,03 & 22,20 \\
N-3 & 11,36 & 10,76 \\
N-6 & 6,35 & 4,43 \\
N3/N6 & 1,78 & 2,63 \\
EPA + DHA & 5,36 & 4,17 \\
EPA / DHA & 0,83 & 3,10 \\
\hline
\end{tabular}

$\mathrm{N}-3$, soma dos ácidos graxos n3 da tabela 1, inclusive EPA e DHA.

N-6, soma dos ácidos graxos n6 da tabela 1 .

artrite e câncer. Dessa forma o consumo de peixe, de óleos de pescado e de concentrados de ácidos graxos tem sido recomendado na alimentação humana.

A dieta alimentar de carpas com dejetos suínos exerce um efeito significativo na composição do tecido muscular, principalmente da composição mineral e de lipídios totais. A alimentação com dejetos suínos produziu carpas com índices menores de lipídios totais e, por conseqüência, teores menores de ômega3, embora carpas, independentemente da alimentação, não representem fonte significativa de EPA e DHA.

\section{CONCLUSÕES}

A composição centesimal do tecido muscular de carpas (Cyprinus carpio) alimentadas com ração e com dejetos suínos foi diferente. As principais diferenças foram observadas no conteúdo de minerais e de lipídios totais, embora a variação observada nos valores de lipídios totais não tenha se refletido no perfil de ácidos graxos.

\section{AGRADECIMENTOS}

Ao Programa Institucional de Bolsas de Iniciação Científica/Conselho Nacional de Pesquisa e ao Fundo de Apoio à Pesquisa/Universidade do Oeste de Santa Catarina, pelo financiamento da pesquisa.

\section{REFERÊNCIAS}

AOAC - Official Methods of Analysis of AOAC International. 16.ed. Whashington, DC, 2000. V.1, Z, 1094p.
BARRETO, C.M. et al. Desempenho de ovinos em terminação com dietas contendo deferentes níveis de dejetos suínos. Revista Brasileira de Zootecnia, v.33, n.6, p.1858-1865, 2004a.

BARRETO, C.M. et al. Productive performance of confined finishing sheep supplemented with different levels of swine waste. Revista Brasileira de Zootecnia, v.33, n.6, p.18581865, 2004b.

BLIGH, E.G.; DYER, W.J. A rapid method of total lipid extraction and purification. Canadian Journal Biochemistry and Physiology, v.27, p.911-917, 1959.

CAVAlCANTI, S.S. Produção de suínos. Campinas, SP: Instituto Campineiro de Ensino Agrícola, 1984. 1 45p.

DIGGS, B.G. et al. Resume value of pig faces in swine finishing rations. Journal of Animal Science, v.24, p.291, 1965.

GURGEL, J.J.S.; FREITAS, J.V.F. Sobre a composição química de doze espécies de peixe de valor comercial de açudes do Nordeste brasileiro. Boletim Técnico, DNOCS, v.30, n.1, p.45-57, 1972.

IBGE - INSTITUTO BRASILEIRO DE GEOGRAFIA E ESTATÍSTICA. Banco de Dados Agregados. Pesquisa do Efetivo de rebanho por tipo de rebanho suíno, 2004. Tabela 73. Capturado em 18 abril 2006. Online. Disponível na Internet: h t t p : / / w w w.sidra.ibge.gov.br/bda/tabela / protabl.asp? $\mathrm{z}=\mathrm{t} \& \mathrm{o}=20 \& \mathrm{i}=\mathrm{P}$.

KING, I. et al. Shellfish: proximate composition, minerals, fatty acids, and sterols. Journal of the Dietetic Association, v.90, n.5, p.667-685, 1990.

KOOP, E.I. Dejetos suínos como componentes de rações juvenis de pacu (Piaractus mesopotomicus Hoimebeg). 1996. 71f. Dissertação (Mestrado em Aquicultura) - Universidade Federal de Santa Catarina.

KRZYNOWERK, L.; PANUNZIO, L.J. Cholesterol and fatty acids in several species of shrimp. Journal of Food Science, v.54, n.2, p.237-239, 1989.

MAIA, E.L. Fatty acids of total, neutral, and phospholipids of Brazilian freshwater fish Prochilodus scrofa. Journal of Food Composition and Analysis, v.7, p.240-251, 1994.

MAIA, E.L. et al. Proximate, fatty acid and amino acid composition of the brazilian freshwater fish prochilodus scrofa. Food Chemistry, v.12, p.275-286, 1983.

MAYSER, P. et al. Omega-3 fatty acid-based lipid infusion in patients with chronic plaque psoriasis: results of a double-blind, randomized, placebo-controlled, multicenter trial. Journal of American Academy Dermatology, v.38, p.421-424, 1998. 